$J$ Am Chem Soc. 2016 February 24; 138(7): 2134-2137. doi:10.1021/jacs.5b13041.

\title{
Oxy-Allyl Cation Catalysis: An Enantioselective Electrophilic Activation Mode
}

\author{
Chun Liu, E. Zachary Oblak, Mark N. Vander Wal, Andrew K. Dilger, Danielle K. Almstead, \\ and David W. C. MacMillan * \\ Merck Center for Catalysis, Princeton University, Princeton, New Jersey 08544, United States
}

\begin{abstract}
A generic activation mode for asymmetric LUMO-lowering catalysis has been developed using the long-established principles of oxy-allyl cation chemistry. Here, the enantioselective conversion of racemic $a$-tosyloxy ketones to optically enriched $a$-indolic carbonyls has been accomplished using a new amino alcohol catalyst in the presence of electron-rich indole nucleophiles. Kinetic studies reveal that the rate-determining step in this $\mathrm{S}_{\mathrm{N}} 1$ pathway is the catalyst-mediated $a$ tosyloxy ketone deprotonation step to form an enantiodiscriminant oxy-allyl cation prior to the stereodefining nucleophilic addition event.
\end{abstract}

Over the past two decades, the field of asymmetric organocatalysis has grown at a rapid pace, fueled largely by the development of generic modes of substrate activation. Indeed, electrophile activation via hydrogen bonding ${ }^{1}$ or iminium catalysis ${ }^{2}$ has provided more than 60 novel transformations that allow for the enantioselective construction of $\mathrm{C}-\mathrm{C}, \mathrm{C}-\mathrm{X}$, and $\mathrm{C}-\mathrm{H}$ bonds. In more recent years, urea or thiourea-mediated anion binding has found widespread utility for the generation of enantiodifferentiated ion pairs, another LUMOlowering platform that offers a vast array of unique applications. ${ }^{3}$ HOMO-raising asymmetric induction has been widely accomplished with enamine catalysis, an activation mode that enables aldehydes and ketones to readily undergo $a$-carbonyl functionalization with a variety of electrophilic coupling partners. ${ }^{4}$ The utility of enamine catalysis is readily appreciated given the prevalence of $a$-aromatic, $a$-aminated, and $a$-oxygenated carbonyls within the fields of pharmaceutical, fine chemical, and natural product synthesis. With this consideration in mind, we recently questioned whether enantioselective $a$-carbonyl functionalization might be accomplished using LUMO-lowering catalysis, specifically founded upon a new oxy-allyl cation activation mode. As a critical design element, we recognized that the use of a LUMO-lowering pathway would allow for the implementation of nucleophile coupling partners in lieu of electrophiles, a strategy that should enable a

\footnotetext{
*Corresponding Author: dmacmill@ princeton.edu.

The authors declare no competing financial interest.

Supporting Information

The Supporting Information is available free of charge on the ACS Publications website at DOI: 10.1021/jacs.5b13041.

Crystallographic data for $\mathrm{C}_{21} \mathrm{H}_{20} \mathrm{BrNO}_{2}$ (CIF)

Crystallographic data for $\mathrm{C}_{66} \mathrm{H}_{74} \mathrm{~N}_{2} \mathrm{O}_{10}$ (CIF)

Experimental procedures and spectral data (PDF)
} 
dramatic increase in the structure and scope of $a$-carbonyl products that would be accessible using asymmetric organocatalysis.

Oxy-allyl cations have been long known as transient electrophilic species since they were first employed as intermediates in the Favorskii rearrangement in $1894 .{ }^{5}$ Since that time, they have also been used as an activation mode for [4+3] cycloadditions ${ }^{6}$ and Nazarov cyclizations $^{7}$ in a variety of natural product syntheses. In 2013, our laboratory found that oxy-allyl cations generated under mild conditions from $a$-tosyloxy ketones (using weak base) do not readily undergo Favorskii contraction and, more importantly, can be trapped by a large array of $\sigma$ - or $\pi$-nucleophiles to directly furnish $a$-heteroaromatic, $a$-aromatic, $a$ aminated, and $a$-oxygenated carbonyl products. ${ }^{8,9}$ Anticipating that this generic mode of $a$ carbonyl activation might be rendered enantioselective, we subsequently sought to find chiral organocatalysts to (a) enable $a$-tosyloxy ketones to readily undergo soft enolization/ fragmentation and (b) subsequently form enantiodiscriminant oxy-allyl cations. Herein we report the successful execution of these ideals and describe a new amino alcohol catalyst that allows the enantioselective coupling of indoles with racemic $a$-tosyloxy ketones.

\section{Results}

As shown in Figure 1, we hypothesized that chiral hydrogen bond-donating catalysts should reversibly bind with $a$-tosyloxy ketones and thereby enable them to undergo softenolization/fragmentation to form enantiodiscriminant oxy-allyl cations. In this vein, we elected to evaluate a variety of amino alcohols given their capacity to function as carbonyl LUMO-lowering catalysts while at the same time being compatible with mildly basic conditions. Preliminary studies from our lab demonstrated that commercially available phenyl prolinol-derived amino alcohol 1 can induce enantiocontrol in the coupling of $a$ tosyloxy cyclopentanone and $\mathrm{N}$-methylindole in the presence of the mild base $\mathrm{K}_{2} \mathrm{HPO}_{4}$ and benzene, albeit with poor levels of selectivity (Table 1, entry 1). ${ }^{10}$ The effect of changing to the bis-naphthyl-substituted catalyst $\mathbf{2}$ and extending the prolinol core to an octahydroindolinol framework (catalyst $\mathbf{3})^{11}$ further improved the enantioselectivity to $55 \%$ and $73 \%$ ee, respectively (entries 2 and 3). We propose that this net increase in enantiocontrol is due to enhanced cation- $\pi$ stabilization between the oxy-allyl cation and the aryl substituents on the catalyst framework. On this basis we next examined the use of perfluorobenzene instead of benzene as the reaction medium, a change that should disfavor any competing cation $-\pi$ interactions between the solvent and the critical oxy-allyl cation intermediate. ${ }^{12}$ Indeed, the perfluorobenzene system exhibited a marked increase in asymmetric induction (entries 3-5). Finally, incorporation of methyl groups at the 1- and 4positions of the naphthalene rings (catalyst 4) increased the selectivity to $92 \%$ ee, while the presence of water further augmented the yield (entry 6, 91\% yield). Control experiments revealed that the presence of the amino alcohol catalyst is critical for the reaction to proceed (entry 7), while the absence of $\mathrm{K}_{2} \mathrm{HPO}_{4}$ severely inhibits the overall efficiency (entry 8, 4\% yield), indicating the soft enolization step requires both a catalyst and mild base.

With optimal conditions in hand, we next examined the scope of this new and enantioselective oxy-allyl cation addition protocol with respect to the indole component. As revealed in Figure 2, this transformation is amenable to changes in both sterics and 
electronics of the nucleophilic partner. ${ }^{10}$ Notably, we were able to extend this oxy-allyl cation activation mode to an iminium cascade sequence that allows the rapid construction of a complex pyrroloindoline architecture, a motif commonly found among a variety of natural product classes. ${ }^{13}$ As revealed in entry 11 , the use of a tryptamine nucleophile with $a$ tosyloxy cyclopentanone in the presence of catalyst 4 leads to facile formation of the corresponding pyrroloindoline in $92 \%$ yield, $84 \%$ ee, and $4: 1 \mathrm{dr}$.

We next evaluated the scope of $a$-tosyloxy ketones (Figure 3). Interestingly, the use of enantioenriched methyl-substituted $a$-tosyloxy cyclopentanones provided an indolic adduct as a single regioisomer with excellent diastereoselectivity (>20:1 dr) using the matched $(S)$ prolinol catalyst (entries 5 and $6,70 \%$ and $62 \%$ respectively). As a useful mechanistic probe, the formation of the identical indole addition adduct is observed from the 3-methyl as well as the 4-methyl-substituted $a$-tosyloxy cyclopentanone system, reaffirming that the transformation proceeds through a common oxy-allyl cation intermediate. ${ }^{10}$

\section{Mechanistic Studies}

To gain a better understanding of the mechanistic details of this new oxy-allyl cation activation mode, initial rate kinetics experiments were performed to elucidate the reaction order for both the ketone and indole coupling partners and for the prolinol catalyst. ${ }^{10} \mathrm{As}$ revealed in Figures 4, 5, and 6, respectively, a first-order dependence in both ketone and organocatalyst and a zero-order dependence in $\mathrm{N}$-methylindole was observed. These experiments are consistent with a pathway wherein the rate-determining step occurs prior to indole addition to the oxy-allyl cation. Moreover, as demonstrated in Scheme 1, a primary KIE of 3.5 was found for $a$-tosyloxy ketones with proton vs deuterium labels at the $a$ carbonyl positions. These studies provide insight that the deprotonation in the initial enolization step is rate-determining. At this stage we cannot state whether the fragmentation/ ionization step happens in concert with the deprotonation event.

Having found that the soft enolization step is rate-determining, we reasoned that there might be an opportunity to demonstrate chemoselectivity for functionalization of $a$-tosyloxy cyclopentanones in the presence of an almost identical aliphatic cyclohexanone derivative. More specifically, we were aware that five-membered cyclic ketones undergo rapid $a$ deprotonation in comparison to their cyclohexanone counterparts due to the unfavorable strain energy that arises from introducing unsaturation into six-membered over fivemembered rings. Indeed, when both ketones were subjected to this asymmetric catalytic protocol in the same vessel, complete selectivity for $a$-tosyloxy cyclopentanone functionalization was observed, yielding $91 \%$ of the smaller ring adduct and nearquantitative recovery of the $a$-tosyloxy cyclohexanone (Scheme 2 ). ${ }^{14}$

Finally, a series of experiments were conducted using $a$ - $\mathrm{Br}$ and $a$-OMs cyclopentanones to discriminate between two possible pathways for asymmetric induction, namely (a) catalyst bound enantiodiscriminant oxy-allyl cation formation and (b) an ionic catalyst-substrate anion-binding activation mode. While the use of different anion leaving groups on the cyclopentyl moiety should have no effect on the enantioselectivity conferred via a common oxy-allyl cation intermediate, we were aware that anion-binding catalysis typically exhibits 
large variations in enantiocontrol as a function of the halide or tosylate leaving group employed. ${ }^{3 \mathrm{e}}$ In the event, the observed selectivities were $92 \%$ and $90 \%$ ee, respectively for the bromo and mesylate groups, strongly suggesting that the catalyst is hydrogen bonded to the oxy-allyl cation in the enantiodetermining step.

Taking into account the combined results of our mechanistic studies, we believe the following catalytic pathway is operative. Hydrogen bonding of amino alcohol 4 to $a$ tosyloxy ketone $\mathbf{1}$ induces a rate-determining deprotonation step with subsequent or concomitant ionization to form the highly reactive cation 2 (Scheme 3). DFT minimization ${ }^{15}$ of the catalyst-bound oxy-allyl cation 2 (DFT-2) suggests that enantiodiscrimination is achieved via shielding of the oxy-allyl cation top face (as shown) by way of a cation- $\pi$ interaction with one of the naphthalene rings on the catalyst framework. Subsequent addition of the indole nucleophile $\mathbf{3}$ to the less sterically encumbered lower face should provide the enantioenriched $\boldsymbol{a}$-heteroaryl ketone $\mathbf{5}^{16}$

\section{Supplementary Material}

Refer to Web version on PubMed Central for supplementary material.

\section{Acknowledgments}

Financial support was provided by NIHGMS (R01 GM078201-05) and gifts from Merck.

\section{References}

1. Miyabe H, Takemoto Y. Carbonyl and Imine Activation. Comprehensive Organic Synthesis (2). Knochel P, Molander GA. ElsevierAmsterdam2014:751.For seminal papers, see: Sigman MS, Jacobsen EN. J Am Chem Soc. 1998; 120:4901.Sigman MS, Vachal P, Jacobsen EN. Angew Chem, Int Ed. 2000; 39:1279.

2. MacMillan DWC. Nature. 2008; 455:304. [PubMed: 18800128] For seminal papers, see: Ahrendt KA, Borths CJ, MacMillan DWC. J Am Chem Soc. 2000; 122:4243.Jen WS, Wiener JJM, MacMillan DWC. J Am Chem Soc. 2000; 122:9874.

3. Brak K, Jacobsen EN. Angew Chem, Int Ed. 2013; 52:534.Zhang Z, Schreiner PR. Chem Soc Rev. 2009; 38:1187. [PubMed: 19421588] For seminal papers, see: Kotke M, Schreiner PR. Tetrahedron. 2006; 62:434.Kotke M, Schreiner PR. Synthesis. 2007; 2007:779.Raheem IT, Thiara PS, Peterson EA, Jacobsen EN. J Am Chem Soc. 2007; 129:13404. [PubMed: 17941641] Reisman SE, Doyle AG, Jacobsen EN. J Am Chem Soc. 2008; 130:7198. [PubMed: 18479086] Zuend SJ, Coughlin MP, Lalonde M, Jacobsen EN. Nature. 2009; 461:968. [PubMed: 19829379]

4. Mukherjee S, Yang JW, Hoffmann S, List B. Chem Rev. 2007; 107:5471. [PubMed: 18072803] For seminal paper, see: Hajos ZG, Parrish DR. J Org Chem. 1974; 39:1615.

5. (a) Favorskii AE. J Russ Phys-Chem Soc. 1894; 26:559.(b) Loftfield RB. J Am Chem Soc. 1951; 73:4707.

6. (a) Harmata M. Chem Commun. 2010; 46:8886.(b) Harmata M. Chem Commun. 2010; 46:8904.

7. West FG, Scadeng O, Wu Y-K, Fradette RJ, Joy S. The Nazarov Cyclization. Comprehensive Organic Synthesis (2). Knochel P, Molander GA. ElsevierAmsterdam2014:827.For examples in recent total syntheses, see: Shvartsbart A, Smith AB. J Am Chem Soc. 2015; 137:3510. [PubMed: 25756504] Shi Y, Yang B, Cai S, Gao S. Angew Chem, Int Ed. 2014; 53:9539.

8. Vander Wal MN, Dilger AK, MacMillan DWC. Chem Sci. 2013; 4:3075. The Chi group concurrently reported a similar transformation: Tang Q, Chen X, Tiwari B, Chi RC. Org Lett. 2012; 14:1922. [PubMed: 22455439] 
9. For other reports employing oxy-allyl, siloxy-allyl, or azaoxy-allyl cations towards racemic electrophilic activation, see: Jeffrey CS, Barnes KL, Eickhoff JA, Carson CR. J Am Chem Soc. 2011; 133:7688. [PubMed: 21534633] Acharya A, Eickhoff JA, Jeffrey CS. Synthesis. 2013; 45:1825.Li H, Hughes RP, Wu J. J Am Chem Soc. 2014; 136:6288. [PubMed: 24739089] Ayala CE, Dange NS, Fronczek FR, Kartika R. Angew Chem, Int Ed. 2015; 54:4641.Dange NS, Stepherson JR, Ayala CE, Fronczek FR, Kartika R. Chem Sci. 2015; 6:6312.Acharya A, Anumandla D, Jeffrey CS. J Am Chem Soc. 2015; 137:14858. [PubMed: 26562215] DiPoto MC, Hughes RP, Wu J. J Am Chem Soc. 2015; 137:14861. [PubMed: 26562355]

10. Se SI for further discussion.

11. A similar catalyst scaffold was employed in the following works: Arceo E, Jurberg ID, AlvarezFernandez A, Melchiorre P. Nat Chem. 2013; 5:750. [PubMed: 23965676] Luo R, Weng J, Ai H, Lu G, Chan ASC. Adv Synth Catal. 2009; 351:2449.

12. (a) Ma JC, Dougherty DA. Chem Rev. 1997; 97:1303. [PubMed: 11851453] (b) Dougherty DA. Science. 1996; 271:163. [PubMed: 8539615]

13. For a review on the synthesis of pyrroloindolines and their reactivity, see: Crich D, Banerjee A. Acc Chem Res. 2007; 40:151. [PubMed: 17309195] For a review of pyrroloindoline-bearing natural products, see: Steven A, Overman LE. Angew Chem, Int Ed. 2007; 46:5488.

14. At the current time, catalyst $\mathbf{4}$ is selective only for five-membered ring ketones; work is ongoing to develop a suitable catalyst for ketones of other ring sizes.

15. DFT optimization used a B3LYP 6-31G basis set with benzene as the solvent.

16. The absolute configuration of the $p$-bromobenzyl derivative of $(S)-5$ was unambiguously determined by X-ray crystallographic analysis, which lends further support for the proposed mechanism; see SI for crystallographic data. 


\section{Established Enantioselective Electrophilic Activation Modes}
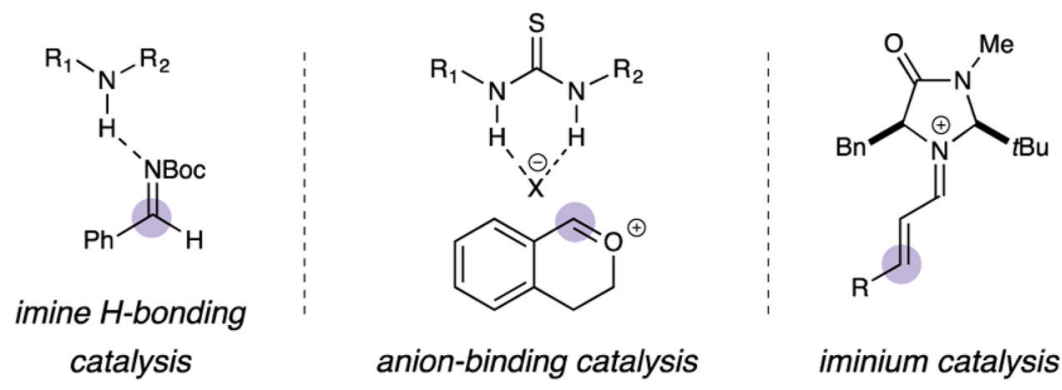

anion-binding catalysis

iminium catalysis

Oxy-Allyl Cation Catalysis: A new platform of electrophilic activation<smiles>O=C1CCCC1Cl</smiles>

racemic ketone<smiles>OCC1C=CCCC1</smiles><smiles>COc1ccccc1</smiles><smiles>C=C</smiles>

enantiodiscriminant oxy-allyl cation

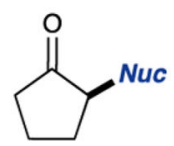

enantioenriched ketone

\section{Diverse Suite of Nucleophilic Reaction Partners}<smiles></smiles>

indoles<smiles>Nc1ccccc1</smiles>

anilines<smiles>C=C([18OH])O[Na]</smiles>

enol silanes<smiles>[14CH3]C(O)[Mg]</smiles><smiles>NC(=O)c1ccccc1</smiles>

amides

Figure 1.

Accessing novel organocatalytic platforms of catalysis. 


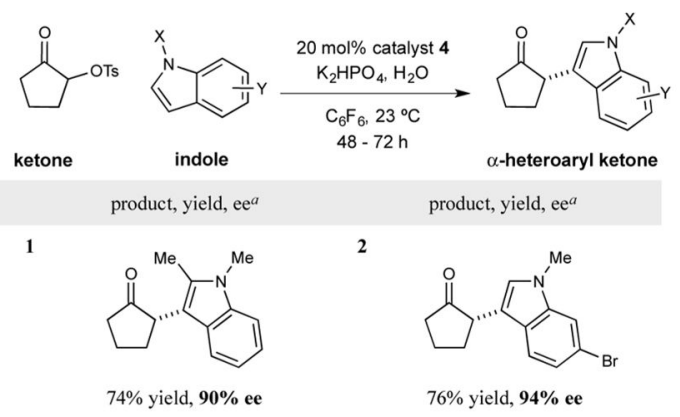

3
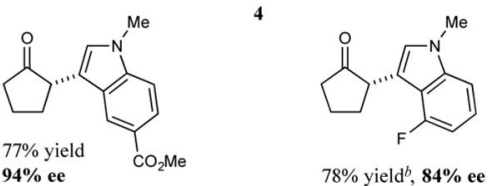

5

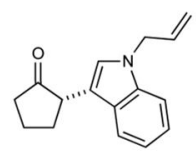

$78 \%$ yield ${ }^{b}, \mathbf{8 4} \%$ ee

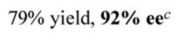

6

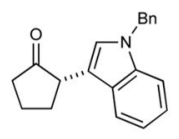

$85 \%$ yield, $92 \%$ ee

7
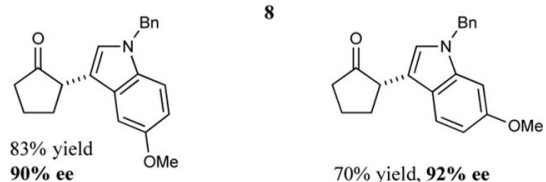

$70 \%$ yield, $\mathbf{9 2} \%$ ee
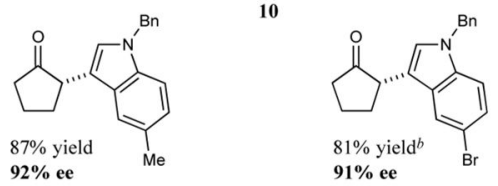

11
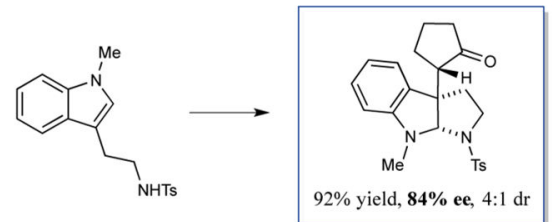

Figure 2.

Scope of the indole nucleophile. ${ }^{a}$ Isolated yields; see Supporting Information (SI) for experimental details. ${ }^{b}$ Reaction time of $96 \mathrm{~h} .{ }^{c}$ Using 1,4-diOMe-2naphthyloctahydroindolinol catalyst. 

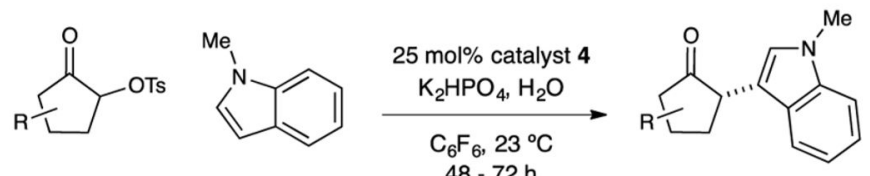

ketone

indole $\mathrm{C}_{6} \mathrm{~F}_{6}, 23^{\circ} \mathrm{C}$
$48-72 \mathrm{~h}$

$\alpha$-heteroaryl ketone

$\alpha$-tosyloxy ketone

product

yield, ee ${ }^{a}$

1
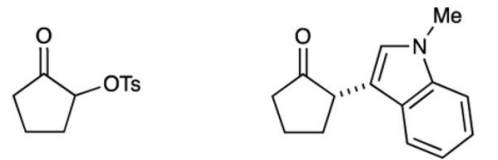

$91 \%$ yield

92\% ee

2<smiles>O=C1CC(Cc2ccccc2)CC1[O+]</smiles><smiles>Cn1cc([C@H]2CC(Cc3ccccc3)CC2=O)c2ccccc21</smiles>

$84 \%$ yield $^{b}$

92\% ee

3<smiles>CCCCCCCCCCCCCCC</smiles><smiles>Cn1cc([C@@H]2CC(C)(C)CC2=O)c2ccccc21</smiles>

yield

$90 \%$ ee

4

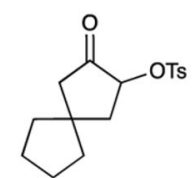

5

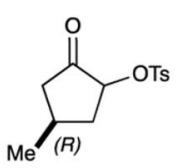

6

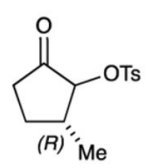<smiles>Cn1cc([C@H]2CC3(CCCC3)CC2=O)c2ccccc21</smiles><smiles>CC1CC(=O)[C@H](c2cn(C)c3ccccc23)C1</smiles><smiles>CC1CC(=O)[C@H](c2cn(C)c3ccccc23)C1</smiles>

$75 \%$ yield $90 \%$ ee

$70 \%$ yield $>20: 1 \mathrm{dr}^{d}$

$54 \%$ yield 2.4:1 dr with ent-cat ${ }^{d}$

$62 \%$ yield $>20: 1$ dr $^{d}$

$46 \%$ yield 3.5:1 dr with ent-cat ${ }^{d}$

Figure 3.

Scope of the ketone electrophile. ${ }^{a}$ Isolated yields; see SI for experimental details. ${ }^{b} 1: 1 \mathrm{dr}$ of product. ${ }^{c}$ Reaction time of 96 h. ${ }^{d}$ Using 1,4-diOMe-2-naphthyloctahydroindolinol catalyst. 


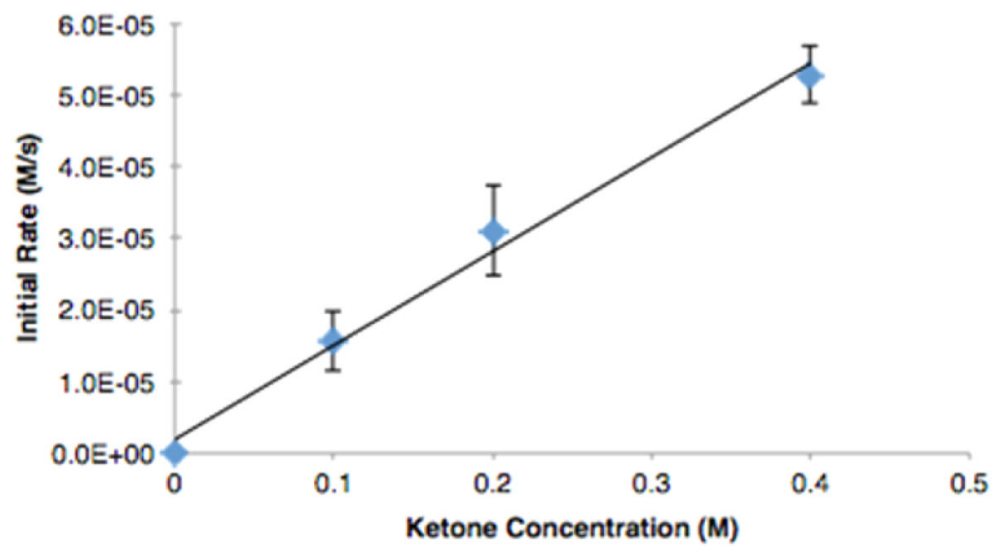

Figure 4.

Plot of initial rate $(\mathrm{M} / \mathrm{s})$ versus [ketone] $(\mathrm{M})$. 


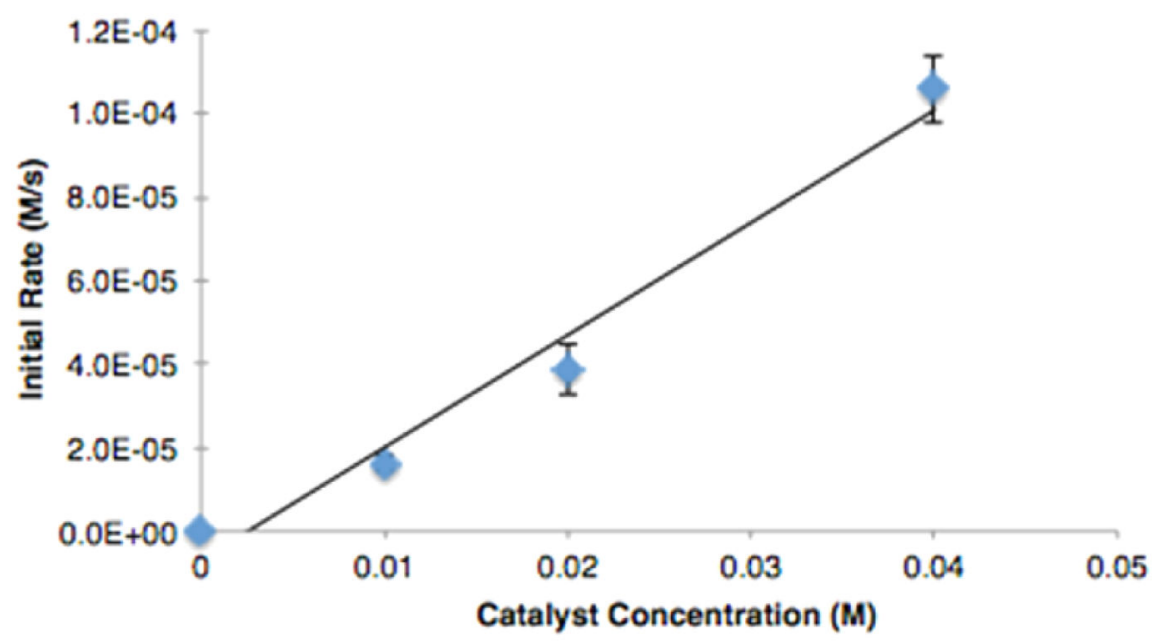

Figure 5.

Plot of initial rate (M/s) versus [organocatalyst] (M). 


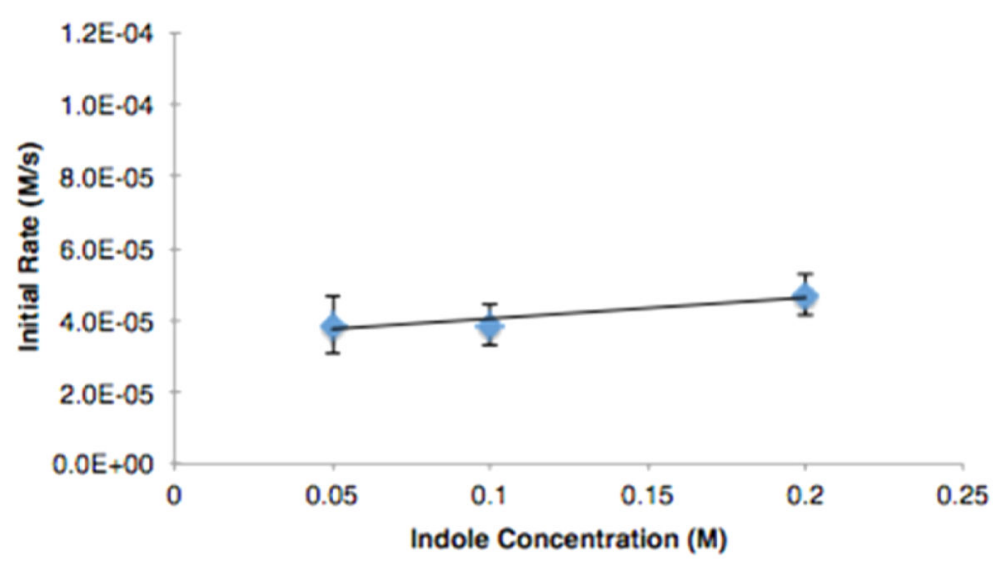

Figure 6.

Plot of initial rate $(\mathrm{M} / \mathrm{s})$ versus [indole] $(\mathrm{M})$. 


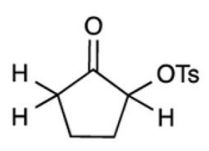<smiles>Cn1ccc2ccccc21</smiles>

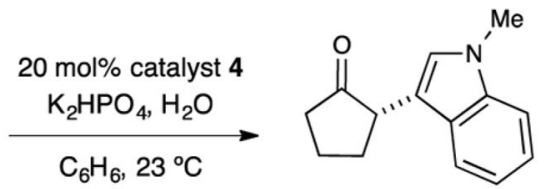<smiles>[2H]C1([2H])CCC([2H])([2H])C1=O</smiles><smiles>Cn1ccc2ccccc21</smiles>

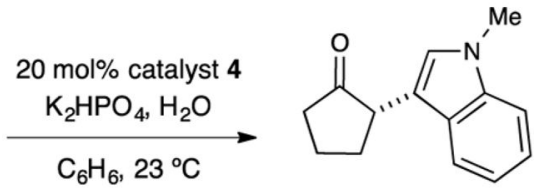

$k_{\mathrm{H}} / \boldsymbol{k}_{\mathrm{D}}=\mathbf{3 . 5}$ - Primary $\mathrm{KIE}, \mathrm{C}-\mathrm{H}$ bond breaking is rate determining

Scheme 1.

Kinetic Isotope Effect Studies on Soft Enolization 

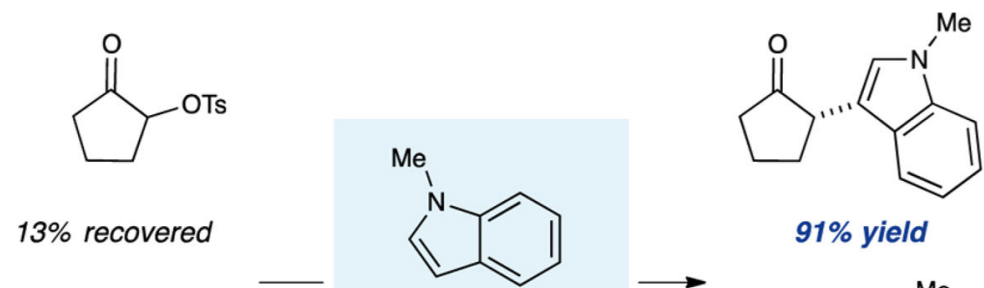

91\% yield

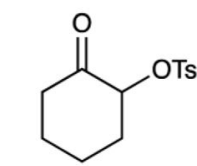

20 mol\% catalyst 4

$91 \%$ recovered

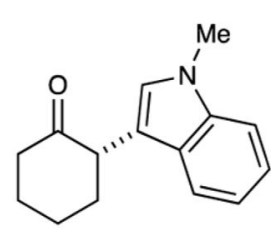

$0 \%$ yield

Scheme 2.

Competition Study Conducted in Common Vessel 
<smiles>O=C1CCCC1[Se+]</smiles><smiles>OC(Br)(Br)[C@H]1C[C@@H]2CCCC[C@H]2N1</smiles>

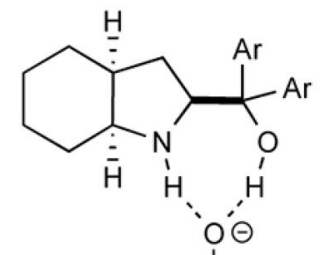

ketone 1

catalyst 4

2
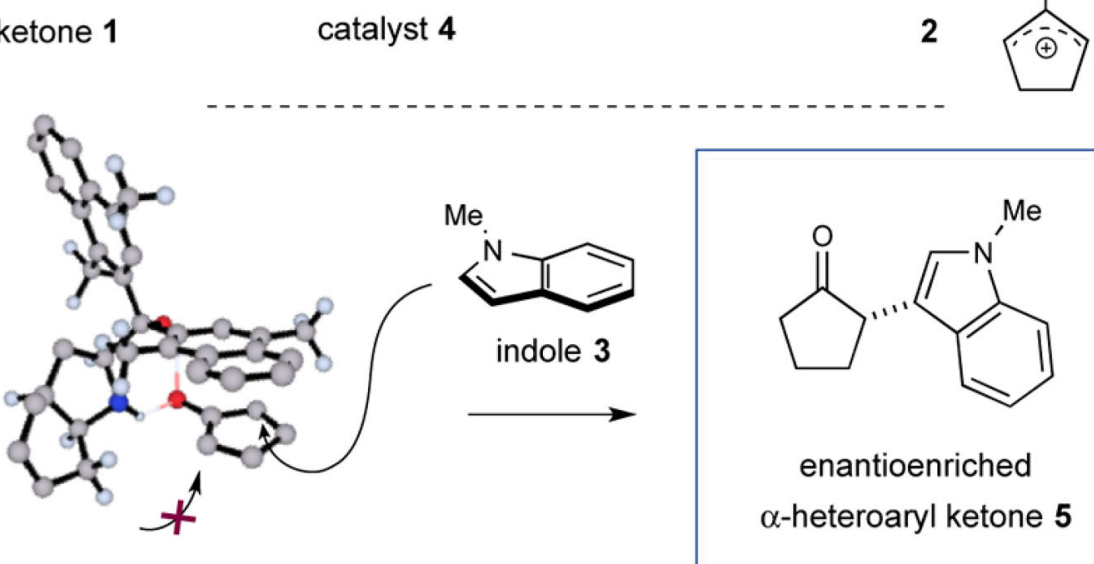

DFT-2

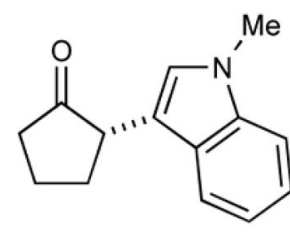

enantioenriched

$\alpha$-heteroaryl ketone 5

Scheme 3.

Proposed Mechanism of the Substitution Reaction 


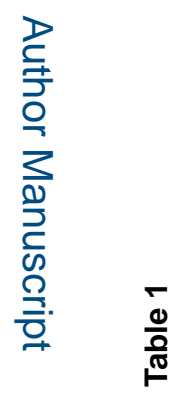

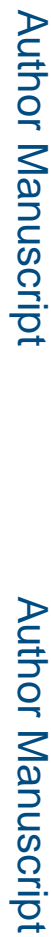

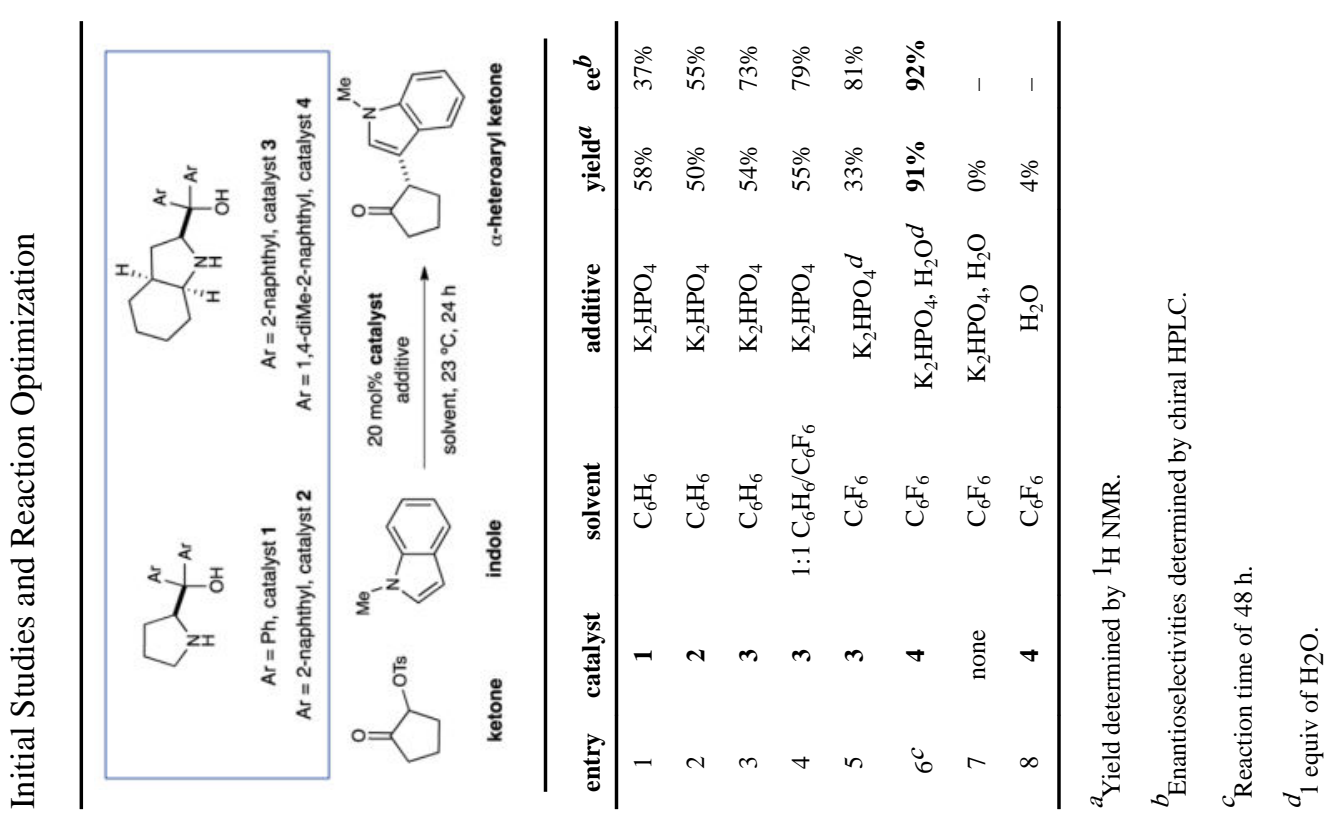

J Am Chem Soc. Author manuscript; available in PMC 2016 November 10. 